\title{
Coulisses
}

Revue de théâtre

6 | Printemps 1992

Varia

\section{Un thème théâtral : Mozart assassiné}

Hélène Lagrange

\section{OpenEdition}

Journals

Édition électronique

URL : http://journals.openedition.org/coulisses/1990

DOI : 10.4000/coulisses.1990

ISSN : 2546-9460

\section{Éditeur}

Presses universitaires de Franche-Comté

\section{Édition imprimée}

Date de publication : 1 juin 1992

ISSN : 1150-594X

\section{Référence électronique}

Hélène Lagrange, «Un thème théâtral : Mozart assassiné », Coulisses [En ligne], 6 | Printemps 1992 mis en ligne le 15 mars 2019, consulté le 30 octobre 2019. URL : http://journals.openedition.org/ coulisses/1990; DOI : 10.4000/coulisses.1990

Ce document a été généré automatiquement le 30 octobre 2019

Coulisses 


\section{Un thème théâtral : Mozart assassiné}

\section{Hélène Lagrange}

Portraits de Salieri
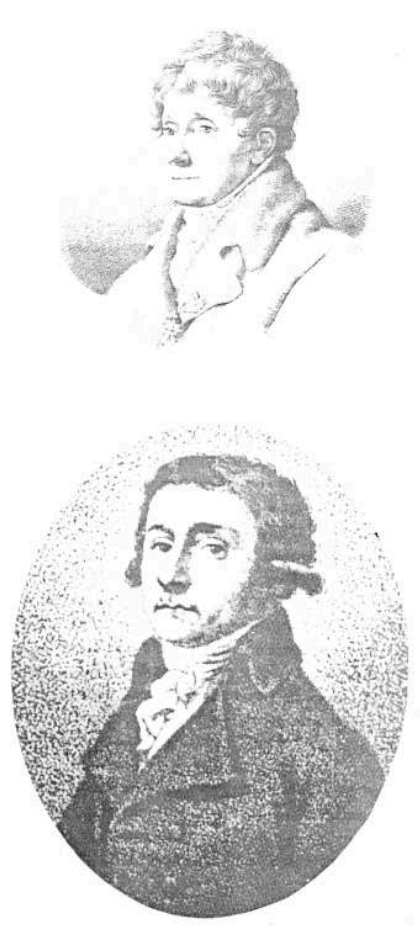

1 Le 2 novembre 1979 était représentée pour la première fois la pièce du dramaturge anglais Peter Schaffer Amadeus au Théâtre National de Londres. La pièce était reprise et représentée un an plus tard le 17 décembre 1980 dans sa deuxième version au Broad Hurst Theater de New-York. L'auteur avait à cœur de nous faire parcourir la vie et l'œuvre de Mozart au travers d'un autre personnage : Antonio Salieri. 
2 Le 27 février 1832, quelques années après la mort de ce compositeur italien né à Legnano en 1750, qui fut successivement compositeur appointé, maître de chapelle impériale et directeur de l'Opéra de Vienne (où il mourut en 1825, Mozart en 1791) était jouée au théâtre de Saint-Pétersbourg une petite tragédie à deux personnages Mozart et Salieri. L'auteur n'était autre que le très grand poète et dramaturge russe Alexandre Sergueïvitch Pouchkine. Si ces deux pièces diffèrent énormément dans leur composition et ont chacune leur originalité, il n'en est pas moins vrai que la trame et la source d'inspiration de chacune reposent sur le personnage de Salieri, autour duquel naquit le mythe d'un Mozart assassiné, empoisonné.

3 Si Salieri vénéra l'œuvre de Gluck et fut l'ami de Haydn, nous savons qu'il fut beaucoup moins celui de Mozart et de Schubert. Les musiciens n'étaient que des serviteurs qui n'échappaient pas à la critique et aux sévères jugements. Mozart, ses amis et ennemis, furent bien du nombre. Ainsi Caroline Pichler, la fille d'un conseiller aulique à Vienne où Mozart faisait jouer ses quatuors, apparaît dans sa correspondance profondément convaincue "de l'inculture » des musiciens en général et de celle en particulier de Mozart, Schubert et Haydn. Ces musiciens étaient aussi des hommes, parfois amis, parfois rivaux, très vite accusés de malveillance. Les rumeurs allaient bon train.

4 L'année même de la mort de Salieri, se répandit largement la nouvelle qu'il aurait avoué avoir empoisonné Mozart. Ses amis s'insurgèrent contre ce fait, crièrent à la calomnie, considérant cet aveu avant tout comme le résultat du délabrement psychique qui assombrit ses dernières années. D'autre part, les maux dont souffrait Mozart et que les médecins de l'époque ne pouvaient pas définir, étaient assez comparables à ceux d'un empoisonnement.

5 En toute objectivité, il faut aussi remarquer que Salieri de son vivant connut autant la gloire que Mozart. Par exemple, en 1786, son Singspiel Prima La Musica E Por Parole est donné en même temps que L'Impresario de Mozart. Toutefois, cette confession ne laissa pas Pouchkine indifférent et il y trouva le sujet d'un drame qui représentait pour lui une aventure étrange, sortant de l'ordinaire, destiné à un public populaire. Il est néanmoins curieux de lire écrit de la main même de Pouchkine, au début des années trente, ces notes : «A la première représentation de Don Juan, alors que le théâtre est plein de spectateurs avertis et émerveillés, écoutant avec vénération la musique harmonieuse de Mozart, quelqu'un siffla - tout le monde se retourna, étonné et indigné, et le célèbre compositeur Salieri sortit de la salle, rongé par la fureur et l'envie ».

6 Certains journaux allemands racontèrent qu'au moment de sa mort, Salieri aurait avoué un crime horrible, l'empoisonnement du grand Mozart. «Celui qui a pu empoisonner Don Juan, conclut Pouchkine, a pu empoisonner son créateur ». A-t-il cru à ces bruits?

7 La pièce de Pouchkine Mozart et Salieri, la seule qui ait été représentée de son vivant, ne fut jouée que deux fois et dans des conditions déplorables, "pour l'assemblée des carrosses » disaient les Russes, c'est-à-dire en pièce sacrifiée. On avait l'habitude pour débuter un spectacle de jouer de méchants vaudevilles qui se débitaient dans le brouhaha des arrivées et que personne n'écoutait.

8 Du point de vue strictement dramatique, on peut reprocher à cette pièce ses longs monologues - qui rendent le rôle de Salieri difficile à tenir et exigent du spectateur un effort d'attention trop soutenu - le caractère sévère de l'œuvre, dû à l'absence totale de 
personnages féminins, et à l'austérité des développements. Il reste que le thème de l'envie est ici porté à la scène avec une rare puissance. Dès le premier long monologue de Salieri, on trouve la peinture de cette passion, contrastant avec l'insouciance de Mozart, une insouciance qui annonce celle de son Don Juan, l'analyse aiguë des conditions de l'activité créatrice, technique laborieuse d'une part, don inné d'une inspiration généreuse d'autre part. On y retrouve aussi les traits familiers des personnages pouchkiniens : le caractère absolu de la passion, le désir d'être le premier, le mépris des prodigalités de la jeunesse.

Entre Mozart. Il vient en ami inviter Salieri à l'auberge. Il se veut léger, insouciant. Il veut chasser une inquiétude : «Un homme en noir est venu commander un requiem ! J'ai honte de l'avouer. Mon homme en noir ne me laisse en repos ni le jour ni la nuit. Il me poursuit partout, comme une ombre ». Une autre ombre le menace, celle de Salieri. Et c'est à lui qu'il se confie, qu'il fait entendre les premières notes de son Requiem, lui qui bientôt va verser du poison dans son verre. L'aveu de sa haine, de sa jalousie précède l'acte criminel.

10 Chez Peter Schaffer, l'aveu est une confession qui permet le retour en arrière et nous situe en leur temps nos deux personnages tout au long de leur vie avec beaucoup de richesse et d'originalité. Le don de la Musique, Salieri le demande à Dieu contre le renoncement aux plaisirs : "Seigneur, laissez-moi devenir un compositeur. Donnezmoi la gloire, et je vous honorerai par ma musique tous les jours de ma vie ». Il se croit exaucé.

J'étais le plus jeune célèbre musicien du temps. Mais voilà que soudain sans crier gare, Mozart.

Et toute sa colère se retourne à la fois contre Dieu et Mozart :

J'ai été bafoué, vous êtes mon ennemi. Je t'ai nommé maintenant toi, Dieu, ennemi immortel et ceci je le jure.

Il voudra se venger.

Ce fut une guerre avec Dieu au travers de la créature qu'il a préférée.

13 Il ne peut supporter de tomber dans l'oubli et voir la gloire de Mozart grandir.

Sa musique a résonné de plus en plus fort à travers le monde, la mienne s'est affadie. Plus personne ne la joue. Non, je ne peux l'accepter. Je n'ai pas vécu sur terre pour avoir été un jouet entre les mains de Dieu. Je veux que l'on se souvienne de moi, si ce n'est pour la gloire, alors pour l'infamie... Aies pitié Mozart. Pardonne à ton assassin. Et maintenant mon dernier acte - une fausse confession courte et convaincante.

14 Ainsi se déclare-t-il son assassin pour connaitre avec lui l'immortalité. Chez Pouchkine, le meurtre est réel et Salieri s'en justifie :

Non - Je ne veux plus lutter contre ma destinée. Je suis choisi pour lui barrer la route, sans quoi nous sommes perdus, nous tous, les serviteurs de la musique et non moi seul, avec ma pauvre gloire... Mozart disparu, l'art dégénérera... Semblable à quelques chérubins, d'en haut il est venu nous faire entendre des chants paradisiaques pour s'envoler après avoir fait dans nos cœurs, à nous, créatures de poussière, palpiter le désir, mais un désir sans ailes.

Ce qu'il a voulu, c'est tuer le génie. Mais au-delà de ces différences, nous retrouvons dans la pièce de Peter Schaffer les mêmes thèmes :

- l'amour précoce et passionné de la musique : «Déjà à l'âge de dix ans certaines bouffées d'accords me donnaient le vertige à en défaillir »

- la musique comme art suprême, art divin 
- le refus des plaisirs : «Jusqu'à ce jour, j'ai vécu dans la vertu et la rigueur »

- le désir de s'élever au rang des grands compositeurs : "Mon seul désir était de rejoindre tous les compositeurs qui avaient célébré sa gloire »

- le contraste du travail laborieux et du don naturel : «J'ai travaillé, j'ai façonné et refaçonné le talent que vous m'avez accordé ».

16 A l'origine de son dépit et de son envie, il y a Mozart, "Mozart méprisant, ricanant, prétentieux, infantile, qui n'a jamais travaillé une minute pour aider un autre homme et vous l'avez choisi ».

17 Cette envie qui prend possession de tout son être en fait un véritable personnage dramatique. Le caractère extrême et absolu de cette passion n'a pas laissé indifférents d'autres grands maîtres de la musique, de la peinture et du cinéma. C'est ainsi que le Mozart et Salieri de Pouchkine inspira à Rimski-Korsakov, un des membres du célèbre "Groupe des Cinq ", un opéra du même nom, au peintre Vroubel son œuvre intitulée Salieri versant du poison à Mozart. Cet artiste russe (1856-1910) se tourna essentiellement vers les thèmes de la littérature et du fantastique. Enfin, plus près de nous, se passionna pour ce sujet le cinéaste Milos Forman qui manifesta très vite après les représentations de la pièce de Peter Schaffer aux Etats-Unis, son désir de collaborer avec lui pour produire son film Amadeus.

Salieri versant du poison à Mozart, tableau de Vroubel

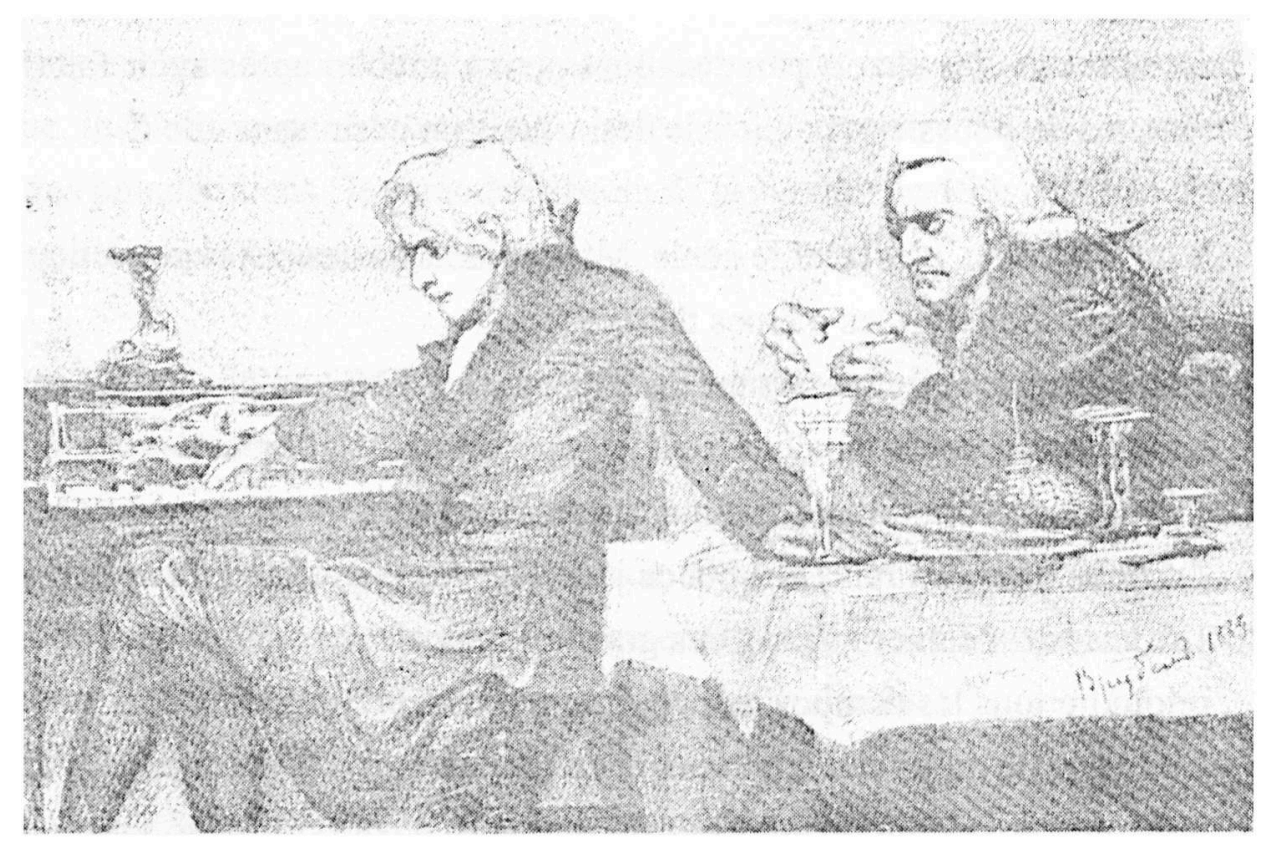

